\title{
Isolation of GFP-tagged plasma membrane protein from Arabidopsis egg cells
}

\author{
Tomoko Igawa ${ }^{1 *}$, Lixy Yamada ${ }^{2}$, Hitoshi Sawada², Toshiyuki Mori ${ }^{3}$ \\ ${ }^{1}$ Graduate School of Horticulture, Chiba University, 648 Matsudo, Matsudo, Chiba 271-8510, Japan; ${ }^{2}$ Sugashima Marine \\ Biological Laboratory, Graduate School of Science, Nagoya University, Sugashima, Toba, Mie 517-0004, Japan; ${ }^{3}$ Department \\ of Tropical Medicine and Parasitology, Juntendo University, 2-1-1 Hongo, Bunkyo-ku, Tokyo 113-8421, Japan \\ *E-mail: tigawa@chiba-u.jp Tel \& Fax:+81-47-308-8853
}

Received March 14, 2017; accepted May 22, 2017 (Edited by S. Nonaka)

\begin{abstract}
Angiosperms possess a double fertilization system for sexual reproduction. Double fertilization is regulated by interactions among proteins localized in the plasma membrane of each sex gamete. A few plasma membrane resident proteins regulating double fertilization have been identified in male gametes. In contrast, no fertilization regulators in female gamete plasma membrane have been identified, largely due to difficulties in the isolation and collection of female gametes. We had produced Arabidopsis transgenic plant pDD45::GFP-AtPIP2;1 where the egg cell plasma membrane was specifically labeled with GFP (Igawa et al. 2013). The protein extract derived from approximately 200 pistils, which contained unfertilized and mature egg cells, was subjected to immunoprecipitation using anti-GFP antibody. As a result, both GFP and AtPIP2;1 were specifically detected in immunoprecipitated proteins from pistil tissues of pDD45::GFP-AtPIP2;1 transgenic plant, but not in those of wild type pistils. It was revealed that specific proteins expressed in the egg cells were successfully isolated from pistil cell population. The method described here showed the feasibility of isolating specific egg cell plasma membrane protein without gamete isolation and collection procedures.
\end{abstract}

Key words: egg cell, immunoprecipitation, plasma membrane protein.

Double fertilization is a unique sexual reproduction system specific to angiosperms, which have flourished and diversified in the process of evolution. A functional megaspore undergoes three rounds of karyokinesis followed by mitosis. After cellularization, dimorphic female gametes, an egg cell and a central cell, are formed in an embryo sac. A microspore undergoes two types of mitosis, giving rise to two sperm cells as the male gametes. The sperm cells develop as a connected pair in a pollen grain or a growing pollen tube after pollination. The pollen tube grows in the pistil and its tip ruptures once it reaches the embryo sac. As a result, a pair of sperm cells is discharged into the boundary of the egg cell and the central cell. After the male and female gametes meet, double fertilization, where one sperm cell fertilizes the egg cell and another sperm cell fertilizes the central cell, occurs in the embryo sac. The fertilized egg cell and central cell develop into an embryo and an endosperm, respectively, and the ovule becomes a seed. A full comprehension of the molecular mechanisms regulating double fertilization is important for introducing innovations in plant propagation and breeding technologies. Double fertilization is regulated by different proteins localized in the gamete plasma membrane of each sex. Two sperm cell specific plasma membrane proteins, GEX2 (GAMETE EXPRESS 2) and GCS1/HAP2 (GENERATIVE CELL SPECIFIC 1/HAPLESS 2), have been identified as fertilization regulators probably involved in attachment and fusion to female gametes during double fertilization, respectively (Mori et al. 2006; Mori et al. 2014; Mori and Igawa 2014; von Besser et al. 2006). GCS1/HAP2 was identified by differential display PCR analyses using isolated generative cells (precursor of sperm cells) of Lilium longiflorum, because an efficient method to isolate and collect the generative cells from pollen protoplasts by Percoll $^{\mathrm{TM}}$ density gradient centrifugation had been established (Tanaka 1988). GEX2 was discovered as an expressed gene in the sperm cell based on the EST library, which was constructed from maize sperm cells isolated by combination of Percoll ${ }^{\mathrm{TM}}$ purification and fluorescence-activating sorting (Engel et al. 2003, 2005). These discoveries were brought about by the isolation and collection of male gametes. In contrast, efficient methods for the isolation and collection of female gametes have not been established. Nevertheless, several

Abbreviations: GFP, green fluorescent protein; AtPIP, Arabidopsis thaliana plasma membrane intrinsic protein; GEX2, gamete expressed 2; GCS1, generative cell specific 1; HAP2, hapless 2; EC1, egg cell 1; Col-0, Colombia-0.

This article can be found at http://www.jspcmb.jp/

Published online June 27, 2017 
omics analyses of female gametes collected by manual isolation from an embryo sac after enzymatic treatment or by microdissection have been reported in Gramineae, Torenia, and Arabidopsis (Abiko et al. 2013; Lê et al. 2005; Okuda et al. 2009; Sprunck et al. 2005; Uchiumi et al. 2007; Wuest et al. 2010). Through these studies, some genes or proteins specifically expressed in female gametes were identified. EC1 (EGG CELL 1) was first found in transcriptomics data of isolated wheat egg cells (Sprunck et al. 2005), and a subsequent study reported that Arabidopsis EC1 orthologues were indirectly involved in double fertilization. EC1 was specifically synthesized in the egg cell and secreted when sperm cell reached the female gametes, to induce the relocation of GCS1/HAP2 molecules from the sperm endomembrane system to the surface (Sprunck et al. 2012). EC1 is the first female fertilization regulator to be identified; however, no female gamete specific plasma membrane proteins directly regulating double fertilization have been identified, mainly because of inaccessibility to female gametes and technical difficulties of isolating large quantity of them.

Tamura et al. reported the isolation of GFP (green fluorescent protein)-tagged nucleoporin comprising the nuclear pore complex by immunoprecipitation using anti-GFP antibody from seedlings expressing RAE1-GFP, which localized to the nuclear envelope (Tamura et al. 2010). This has prompted us to look into the possibility of whether angiosperm female membrane protein complex can be isolated in a similar manner without gamete isolation or not. Previously, we produced Arabidopsis transgenic plant pDD45::GFP-AtPIP2;1 where the fusion gene of GFP and AtPIP2;1 (Arabidopsis thaliana aquaporin) is driven under the control of egg cell specific DD45 (a member of EC1 family) promoter (Igawa et al. 2013; Igawa and Mori 2013; Sprunck et al. 2012; Steffen et al. 2007). In this marker line, egg cell plasma membrane is specifically visualized with GFP (Figure 1A) because of AtPIP2;1 distribution. Using this transgenic plant, herein we report a novel method for the isolation of egg cell plasma membrane proteins without gamete isolation.

In order to obtain mature and unfertilized egg cells, emasculation was performed one day before anthesis. The following day, pistils were collected and preserved at $-80^{\circ} \mathrm{C}$ until protein extraction. Because background of pDD45::GFP-AtPIP2;1 transgenic (hereafter called "TG") plant was ecotype Colombia-0 (Col-0), pistils of wild type (WT) Col-0 plants were also used as the experimental control. Protein extraction and immunoprecipitation with anti-GFP antibody were performed using a $\mu$ MACS Epitope Tag Protein Isolation Kit (Miltenyi Biotec, Bergish Gladbach, Germany), in accordance with the protocol reported in the previous study of nucleoporin isolation (Tamura et al. 2010). Approximately $0.1 \mathrm{~g}$ of pistils (roughly corresponding
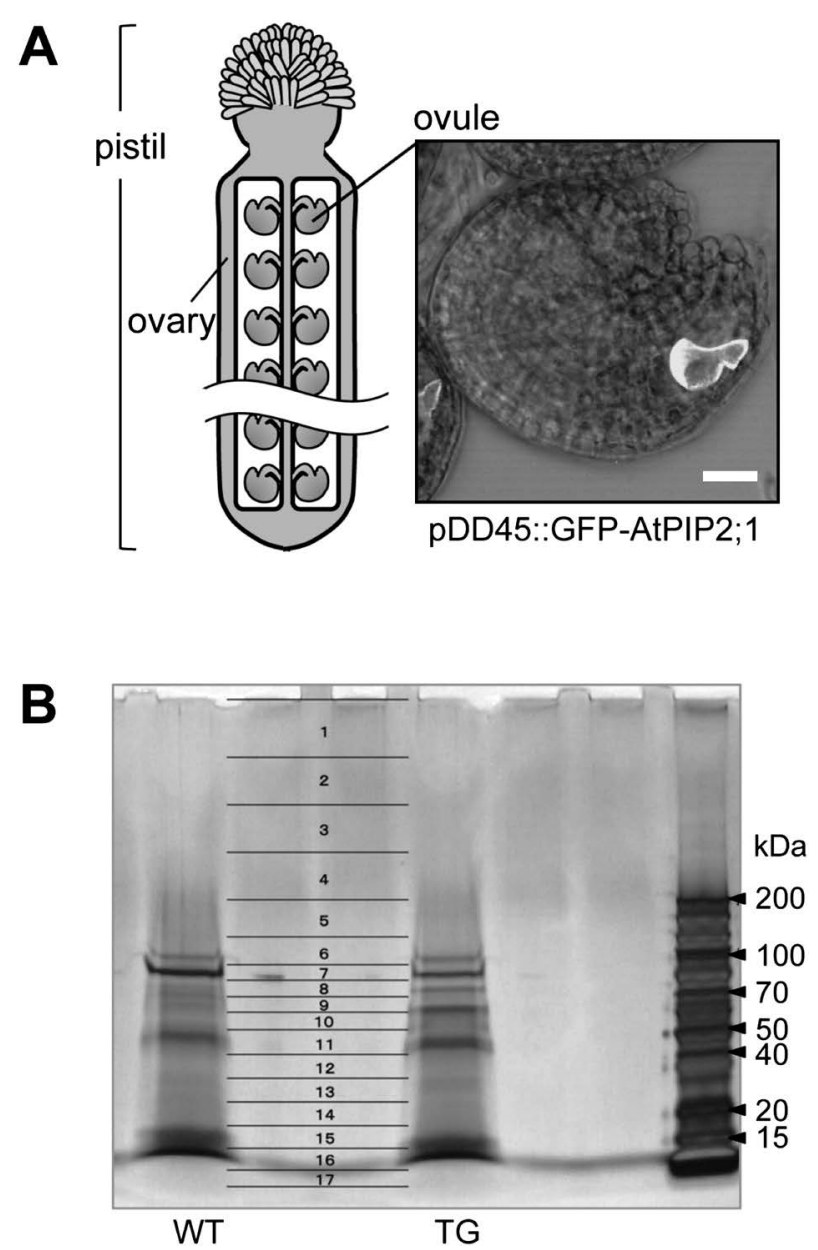

Figure 1. Immunoprecipitation of egg cell membrane proteins (A) One pistil of $A$. thaliana Col- 0 contains 50-60 ovules enclosed by an ovary. In each ovule of pDD45::GFP-AtPIP2;1 transgenic plant, egg cell plasma membrane is specifically labeled with GFP (white intense signal in right image). Bright-field and GFP images were captured with a twophoton laser scanning microscope (FV1000-MPE; Olympus) using a DP-72 digital camera, and the merge of each single-section image is indicated. Bar, $20 \mu \mathrm{m}$. (B) Immunoprecipitated proteins with anti-GFP antibody from WT (wild type Col-0) and TG (pDD45::GFP-AtPIP2;1 transgenic plant) pistils were separated by SDS-PAGE and visualized by silver staining. The gel image in which the Y-axis was magnified by 1.5 times is shown. Each lane of WT and TG was cut into 17 gel slices as shown by fraction numbers. Numbers on the right side of the gel image indicate molecular sizes $(\mathrm{kDa})$ of each band marked with an arrowhead.

to 200 pistils) was ground in $1 \mathrm{ml}$ of lysis buffer $(50 \mathrm{mM}$ HEPES-KOH; pH 7.5, $0.15 \mathrm{M} \mathrm{NaCl}, 0.5 \%$ Triton X-100, and $0.1 \%$ Tween 20 ), and the mixture was incubated on ice for $30 \mathrm{~min}$ with occasional mixing. Then, the mixture was centrifuged at $10,000 \mathrm{~g}$ for $15 \mathrm{~min}$ at $4^{\circ} \mathrm{C}$. The supernatant was subjected to immunoprecipitation. Fifty microliter of anti-GFP MicroBeads included in $\mu$ MACS GFP isolation kit (Miltenyi Biotec) was added to the supernatant and the mixture was incubated for $30 \mathrm{~min}$ on ice. Then the mixture was applied to $\mu$ Column. After washing, the immunoprecipitated proteins were eluted. Isolated immunoaffinity complexes were further subjected to SDS-PAGE using $10 \%$ polyacrylamide gel 
(10\% Mini-PROTEAN ${ }^{\circledR}$ TGX $^{\mathrm{TM}}$ Precast Protein Gel; BioRad, CA, USA), followed by staining with a SilverQuest ${ }^{\mathrm{TM}}$ Silver Staining Kit (Thermo Fisher Scientific Inc., MA, USA). To compare immunoprecipitated proteins from TG pistils and wild type pistils, each lane was sliced into 17 gel fractions in the same manner, although some specific bands were shown in the proteins isolated from TG pistils (Figure 1B). Each gel slice was digested with trypsin to obtain peptides. Digested peptides with trypsin were analyzed by LC-MS/MS; capillary liquid chromatography system (Ultima3000, Dionex) connected online to mass spectrometer (LTQ-XL, Thermo Fisher Scientific Inc.). Acquisition of MS/MS spectra was performed using the parameters reported in the previous study (Yoshida et al., 2014). Raw spectrum data were processed using SEQUEST software to extract peak lists. The obtained peak lists were analyzed using the MASCOT program against database from The Arabidopsis Information Resource (TAIR; http:// www.arabidopsis.org/). Proteins detected from peptide fragments with high reliability [MASCOT score $>40$ ] were selected as identified proteins. LC-MS/MS analysis was performed two times for the same peptide sample from each gel slice. The detected proteins, which were specifically isolated from TG pistils and showed a higher score than GFP, are listed in Table S1.

Notably, polypeptides of both GFP and AtPIP2;1 (AT3G53420) were specifically detected in the immunoprecipitated proteins from TG pistils (Table 1). This result indicates that GFP-tagged plasma membrane protein expressed only in the egg cell was successfully isolated from pistil cell population. Both proteins were detected in \#9-12 gel slices in addition to \#6 gel slice, whereas the putative molecular mass of GFP-AtPIP2;1 was slightly more than $50 \mathrm{kDa}$ (Lee et al. 2009). In a previous study, the monomeric and dimeric forms of AtPIP2;1 were observed by SDS-PAGE (Lee et al. 2009), in contrast to PIPs, which are known to form tetramers in vivo. AtPIP2;1 is also known to be ubiquitinated

Table 1. Detected peptide sequences of GFP-AtPIP2;1 fusion protein.

\begin{tabular}{llrcc}
\hline Protein & Peptide sequence & $\begin{array}{c}\text { Gel } \\
\text { slice* }\end{array}$ & $\begin{array}{c}\text { Frequency of } \\
\text { detection** }\end{array}$ & $\begin{array}{c}\text { Total Score of } \\
\text { each peptide }\end{array}$ \\
\hline AtPIP2;1 & AFQSSYYTR & 6 & 2 & 98.2 \\
& & 11 & 1 & 46.7 \\
& DVEAVPGEGFQTR & 6 & 2 & 143.7 \\
& & 11 & 2 & 127.4 \\
GFP & FEGDTLVNR & 12 & 1 & 41.9 \\
& & 10 & 1 & 42.2 \\
& & 11 & 2 & 41.9 \\
& SAMPEGYVQER & 6 & 2 & 90.5 \\
& & 9 & 1 & 99.7 \\
& & 11 & 1 & 40.8 \\
\hline
\end{tabular}

*: Numbers correspond to gel slices indicated in Figure 1B. **: Number of times detected in two LC-MS/MS analyses.
(Chevalier and Chaumont 2015). The detection of GFP and AtPIP2;1 in \#6 gel slice may imply that these modifications occurred during the isolation procedure. On the other hand, the detection of AtPIP2;1 in \#12 gel slice, which included proteins smaller than GFPAtPIP2;1, may imply the detection of native AtPIP2;1 simultaneously isolated by binding to GFP-AtPIP2;1, or might reflects that truncation occurred. In addition, several proteins specific to TG and showing higher total score (sum of the scores of all peptide matches) than GFP were also identified (Table S1). Among them, AT3G16470 was reported as a GFP-interacting protein (Bellati et al. 2016). Meanwhile, there was no overlap with proteins that interacted with AtPIP2;1 (BioGRID Database for PIP2;1, https://thebiogrid.org/) (Bellati et al. 2016). Therefore, it was suggested that these proteins were isolated because of artificial binding to GFP or AtPIP2;1 during the experiment. However, it could be speculated that AtPIP2;1 interacting proteins were also isolated. Studies have revealed the AtPIP2;1 endomembrane trafficking pathway from ER to plasma membrane, indicating that the subcellular localization of AtPIP2;1 was regulated by phosphorylation and ubiquitination in response to abiotic stress (Chevalier and Chaumont 2015; Lee et al. 2009; Luu and Maurel 2013). According to TAIR descriptions, AT1G52400, AT5G22610, AT1G52030, and AT1G11530, which were identified in this study (Table S1), are involved in abiotic stress response or ubiquitination. It implies that these are the AtPIP2;1-interacting proteins involved in membrane trafficking in the egg cell, although the interaction among these proteins and their roles need to be confirmed experimentally in the future.

In this study, GFP-tagged plasma membrane protein from egg cell was successfully isolated from a protein population derived from pistil tissue. This method is advantageous in that laborious work for the isolation of female gametes from each embryo sac can be omitted. One pistil of $A$. thaliana Col-0 contains 50-60 ovules and approximately 200 pistils were used in the immunoprecipitation experiment. Therefore, 10,000 egg cells at the lowest estimate within the pistil cell population enabled the isolation of the specific plasma membrane protein tagged with GFP in this study. As the immunoprecipitation of GFP-tagged nucleoporin also enabled the identification of proteins forming the nucleoporin complex (Tamura et al. 2010), we expected that proteins binding to the targeted egg cell plasma membrane protein would be isolable by our method. In addition, it was reported that cross-link of protein complexes by formaldehyde treatment allowed isolation of many AtPIP2;1 interacting membrane proteins from whole plants expressing 35Spro::GFP-AtPIP2;1 (Bellati et al. 2016). Application of the known male fertilization regulators such as GCS1/HAP2 and GEX2 fused to 
GFP, and addition of crosslinking step likely enable identification of the interacting proteins localizing to the female gamete plasma membrane. Regarding to GCS1/HAP2, it has been reported that GCS1/HAP2 is homologous to viral class II fusion protein (Fédry et al. 2017; Pinello et al. 2017; Valansi et al. 2017). To achieve somatic cell fusion of mammalian cells, ectopic expression of Arabidopsis GCS1/HAP2 was required for both cells, indicating bilateral homotypic mechanism of GCS1/HAP2. Since GCS1/HAP in flowering plants is specific to male gametes, it has been hypothesized that female gametes express a GCS1/HAP2 family protein that interacts with sperm GCS1/HAP2 as the bilateral mechanism for fertilization (Valansi et al. 2017). When a secretory-type GCS1/HAP2 (named GAH), in which the single transmembrane region of GCS1/ HAP2 was replaced by GFP, was ectopically expressed in Arabidopsis egg cell, occasional blockade of double fertilization was observed (Mori 2014). This phenotype suggested that the antecedent binding of GAH to the unknown GCS1/HAP2-partner protein on the egg cell surface rendered sperm GCS1/HAP2 incapable of binding to its bona fide partner protein. If that suggestion were true, the egg cell surface resident protein directly involved in double fertilization via binding to sperm GCS1/HAP2 would be identified by our method using the GAH-expressing transgenic plant.

\section{Acknowledgements}

We are grateful to S. M. and K. S. for technical assistance. This study was supported by Grants-in-Aid for Scientific Research on Innovative Areas (JSPS) to T.I. (JP22112515), L. Y. (JP22112511), H. S. (JP21112001) and T. M. (JP21112008) and by a Grant-in-Aid for Challenging Exploratory Research to T. I. (JP22657017).

\section{References}

Abiko M, Furuta K, Yamauchi Y, Fujita C, Taoka M, Isobe T, Okamoto T (2013) Identification of proteins enriched in rice egg or sperm cells by single-cell proteomics. PLoS One 8: e69578

Bellati J, Champeyroux C, Hem S, Rofidal V, Krouk G, Maurel C, Santoni V (2016) Novel aquaporin regulatory mechanisms revealed by interactomics. Mol Cell Proteomics 15: 3473-3487

Chevalier AS, Chaumont F (2015) Trafficking of plant plasma membrane aquaporins: Multiple regulation levels and complex sorting signals. Plant Cell Physiol 56: 819-829

Engel ML, Chaboud A, Dumas C, McCormick S (2003) Sperm cells of Zea mays have a complex complement of mRNAs. Plant J 34: 697-707

Engel ML, Holmes-Davis R, McCormick S (2005) Green sperm. Identification of male gamete promoters in Arabidopsis. Plant Physiol 138: 2124-2133

Fédry J, Liu Y, Péhau-Arnaudet G, Pei J, Li W, Tortorici MA, Traincard F, Meola A, Bricogne G, Grishin NV, et al. (2017) The ancient gamete fusogen HAP2 is a eukaryotic class II fusion protein. Cell 168: 904-915

Igawa T, Mori T (2013) Gamete membrane dynamics during double fertilization in Arabidopsis. Plant Signal Behav 8: e24512
Igawa T, Yanagawa Y, Miyagishima S, Mori T (2013) Analysis of gamete membrane dynamics during double fertilization of Arabidopsis. J Plant Res 126: 387-394

Lê Q, Gutièrrez-Marcos JF, Costa LM, Meyer S, Dickinson HG, Lörz H, Kranz E, Scholten S (2005) Construction and screening of subtracted cDNA libraries from limited populations of plant cells: A comparative analysis of gene expression between maize egg cells and central cells. Plant J 44: 167-178

Lee HK, Cho SK, Son O, Xu Z, Hwang I, Kim WT (2009) Drought stress-induced Rma1H1, a RING membrane-anchor E3 ubiquitin ligase homolog, regulates aquaporin levels via ubiquitination in transgenic Arabidopsis plants. Plant Cell 21: 622-641

Luu DT, Maurel C (2013) Aquaporin trafficking in plant cells: An emerging membrane-protein model. Traffic 14: 629-635

Mori T (2014) Profiling the GCS1-Based Gamete Fusion Mechanism. In: Sawada H, Inoue N, Iwano M (eds) Sexual Reproduction in Animals and Plants. Springer Open, London, pp 329-335

Mori T, Igawa T (2014) Gamete attachment process revealed in flowering plant fertilization. Plant Signal Behav 9: e977715

Mori T, Igawa T, Tamiya G, Miyagishima S, Berger F (2014) Gamete attachment requires GEX2 for successful fertilization in Arabidopsis. Curr Biol 24: 170-175

Mori T, Kuroiwa H, Higashiyama T, Kuroiwa T (2006) GENERATIVE CELL SPECIFIC 1 is essential for angiosperm fertilization. Nat Cell Biol 8: 64-71

Okuda S, Tsutsui H, Shiina K, Sprunck S, Takeuchi H, Yui R, Kasahara RD, Hamamura Y, Mizukami A, Susaki D, et al. (2009) Defensin-like polypeptide LUREs are pollen tube attractants secreted from synergid cells. Nature 458: 357-361

Pinello JF, Lai AL, Millet JK, Cassidy-Hanley D, Freed JH, Clark TG (2017) Structure-function studies link class II viral fusogens with the ancestral gamete fusion protein HAP2. Curr Biol 27: 651-660

Sprunck S, Baumann U, Edwards K, Langridge P, Dresselhaus $\mathrm{T}$ (2005) The transcript composition of egg cells changes significantly following fertilization in wheat (Triticum aestivum L.). Plant J 41: 660-672

Sprunck S, Rademacher S, Vogler F, Gheyselinck J, Grossniklaus U, Dresselhaus T (2012) Egg cell-secreted EC1 triggers sperm cell activation during double fertilization. Science 338: 1093-1097

Steffen JG, Kang IH, Macfarlane J, Drews GN (2007) Identification of genes expressed in the Arabidopsis female gametophyte. Plant J 51: 281-292

Tamura K, Fukao Y, Iwamoto M, Haraguchi T, Hara-Nishimura I (2010) Identification and characterization of nuclear pore complex components in Arabidopsis thaliana. Plant Cell 22: 4084-4097

Tanaka I (1988) Isolation of generative cells and their protoplasts from pollen of Lilium longiflorum. Protoplasma 142: 68-73

Uchiumi T, Shinkawa T, Isobe T, Okamoto T (2007) Identification of the major protein components of rice egg cells. J Plant Res 120 : 575-579

Valansi C, Moi D, Leikina E, Matveev E, Graña M, Chernomordik LV, Romero H, Aguilar PS, Podbilewicz B (2017) Arabidopsis $H A P 2 / G C S 1$ is a gamete fusion protein homologous to somatic and viral fusogens. J Cell Biol 216: 571-581

von Besser K, Frank AC, Johnson MA, Preuss D (2006) Arabidopsis HAP2 (GCS1) is a sperm-specific gene required for pollen tube guidance and fertilization. Development 133: 4761-4769

Wuest SE, Vijverberg K, Schmidt A, Weiss M, Gheyselinck J, Lohr M, Wellmer F, Rahnenführer J, von Mering C, Grossniklaus U 
(2010) Arabidopsis female gametophyte gene expression map reveals similarities between plant and animal gametes. Curr Biol 20: 506-512

Yoshida MA, Yamada L, Ochi H, Iwata Y, Tamura-Nakano M,
Sawada H, Sauer WH, Ogura A, Hirohashi N (2014) Integrative omics analysis reveals differentially distributed proteins in dimorphic euspermatozoa of the squid, Loligo bleekeri. Biochem Biophys Res Commun 450: 1218-1224 